\title{
Management of alcohol Korsakoff syndrome
}

\section{Iain Smith \& Audrey Hillman}

\begin{abstract}
"Contrary to popular belief, partial recovery from Korsakoff's Psychosis is the rule and $21 \%$ recover more or less completely. However the extent to which the patient will recover cannot be predicted with confidence during the acute stages of the illness. Failure to appreciate these facts about the natural history of the mental illness may result in the premature confinement of the patient to a mental hospital" (Victor et al, 1971).
\end{abstract}

There is a commonly held view that the Korsakoff syndrome (amnestic syndrome) is a static condition and that, once detected, the main issue in management is suitable placement. However, any decision on long-term placement has to be taken at the point where the potential for further recovery is unlikely and this requires regular and careful review of a patient's mental state during the first year after presentation. There is current concern from such sources as the Mental Welfare Commission in Scotland that patients may be moved at too early a stage to residential care, without adequate psychiatric review, and with poor understanding by the residential or nursing home staff of the nature of this condition. Much of the current literature on the condition focuses on aetiology or on the search for drug treatments for the syndrome. There is very little literature on rehabilitation of individuals with the syndrome in general psychiatric textbooks and journals.

This paper aims to summarise the main medical treatment for Korsakoff syndrome and its complications and to focus on issues of rehabilitation. For all practical purposes we are discussing the management of alcohol Korsakoff syndrome (alcohol amnestic disorder), as cases of non-alcoholic Korsakoff syndrome of chronic duration are exceedingly rare.
The common association of the WernickeKorsakoff syndrome and alcohol dependence in Western societies is well described and any consideration of the management of these patients must include the need to promote abstinence from alcohol and treat the alcohol dependence where this seems possible.

The paper also aims to increase understanding of the spectrum of neuropsychological deficits in this group and knowledge of prognostic indicators for recovery from this disorder. The possible role of rehabilitation psychiatry, a less-resourced speciality in the era of community care, is also discussed.

\section{Definition of the Wernicke- Korsakoff syndrome}

For a full outline of this syndrome, the reader is advised to consult the classic research monograph by Victor et al (1989), and two review articles in the British Journal of Psychiatry (Kopelman, 1995; Lishman, 1990) (see Box 1 for summary points).

Not all cases of Korsakoff syndrome are preceded by an episode of Wernicke's encephalopathy. However, if during an episode of Wernicke's encephalopathy (which is essentially a toxic confusional state) a specific amnesic deficit is detectable, then the condition is likely to proceed to a full Korsakoff syndrome and will respond slowly, if at all, to thiamine supplementation.

In its purest form, Korsakoff syndrome would be an example of an amnestic syndrome, which implies a specific deficit of recent memory with a relatively intact remote memory and intellect. However, in

Iain David Smith is a consultant psychiatrist and Honorary Clinical Senior Lecturer at Gartnavel Royal Hospital (1055 Great Western Road, Glasgow G12 $0 \mathrm{XH}$ ). He is a general psychiatrist with a special interest in alcohol and drug problems. With research interest in the epidemiology of Korsakoff psychosis in Scotland, he has also collaborated in research into possible genetic influences in alcohol-related brain damage. Audrey Hillman is a consultant psychiatrist at Ravenscraig Hospital (Inverkip Road, Greenock, PA16 9HA). Also a general psychiatrist with a special interest in alcohol problems, she works in an area where there is a high prevalence of alcohol-related brain damage and the local service is exploring the possibility of developing a unit specifically for this patient group. 
Box 1. Korsakoff syndrome

Most commonly seen as an enduring complication of the alcohol dependence syndrome

Not always preceded by Wernicke's encephalopathy

Classically is an amnestic syndrome with impaired recent memory and relatively intact intellectual function

Can show varying degrees of recovery

Deficits are open to certain forms of rehabilitative intervention in order to maximise social function

practice, patients will present with a range of features from the pure amnestic syndrome through a mixed picture of amnesia and additional cognitive deficits through to alcohol dementia.

In clinical practice, the terms Korsakoff syndrome and alcohol dementia are often used loosely and interchangeably, perhaps reflecting some of the limitations of bedside mental state examination, (Anonymous, 1987). Precise methods of assessment to classify patients on dimensions of amnesia and dementia are therefore useful in that they may have prognostic and therapeutic implications. This is the opinion of Lishman (1990) who believes that these two dimensions may have differing aetiologies and that in order to develop chronic Korsakoff syndrome, the patient needs to have a combination of thiamine depletion-induced and alcohol-induced brain damage with both sub-cortical and cortical pathologies.

\section{Epidemiology}

Within Scotland the proportion of patients with Korsakoff syndrome and alcoholic dementia in medium- to long-stay psychiatric wards has remained high during a period of progressive bed closures and the trend towards community care. The prevalence of the disorder varies across countries. The incidence of the disorder is increasing in Scotland according to official returns from all hospitals on admissions to in-patient care and also direct ascertainment in general and psychiatric hospitals (Ramayya \& Jauhar, 1997). This is also true in some other European countries, such as The Netherlands where a 20-fold increase in admissions with this diagnosis was found between 1970 and 1990 (Kok, 1991). There is less published information for England and Wales. There is debate in the literature about the reason for this increase. Suggested reasons include increased per capita consumption of alcohol, decreased prescribing of prophylactic parenteral vitamins to 'at-risk alcoholics' in general medical and psychiatric settings, and increased social isolation of alcoholdependent individuals, resulting in increased alcohol consumption and poorer diet (Price, 1985).

The literature suggests there are important gender differences in the presentation and outcome in Korsakoff syndrome. It would appear that women are more vulnerable to developing this syndrome and that cases of Korsakoff syndrome are overrepresented in females if the greater rates of alcohol dependence in men are accepted(Victor et al, 1989).

In the Victor et al series, the age at onset in women was on average a decade earlier than in men. Average age of onset for women was in the fifth decade. This is not reflected in other series and in particular in psychiatric hospital populations. This latter finding may be biased, as younger women may be more likely to be cared for by relatives or to have fewer behavioural difficulties associated with the condition. There is a shorter drinking time before developing Korsakoff syndrome in women. In one study, the mean number of years of heavy drinking for females was half that for males who develop Korsakoff syndrome; $10 v .21$ years, respectively (Cutting, 1978).

Gender differences may influence the prognosis in a more complex way. In the series of 63 patients studied by Cutting (1978), there was a subgroup of females who developed alcohol Korsakoff syndrome a decade later than men. They tended to have insidiously developing Korsakoff syndrome and their recovery tended to be more complete. They also showed a more global impairment of intellect and were comparable to a group labelled 'alcohol dementia'. It would therefore appear that the rate of development of the syndrome is an important prognostic factor in conjunction with gender and age. Sudden onset cases with a dense and relatively pure amnestic syndrome seem to have a poorer prognosis than those with insidious onset and more global impairment (see Box 2).

\section{Assessment}

These patients are often first encountered in general hospital settings or in squalor at home. They are rarely referred directly to general psychiatry. They sometimes present to old age psychiatrists or liaison psychiatry services. The Wernicke-Korsakoff syndrome can be associated with an alcohol- 
Box 2. Prognosis in Korsakoff syndrome

Poorer in sudden-onset than in insidiousonset cases

Better with more global cognitive impairment than in purer amnestic syndrome (provided there is abstinence from alcohol)

Appears better in non-alcoholic cases of Wernicke-Korsakoff syndrome

Improved if abstinence from alcohol is maintained in milder cases

withdrawal delirium in the initial stages (Victor $e t$ al, 1989).

\section{Acute assessment and management}

Ideally, if patients are still showing signs of a global confusional state, they should be nursed in a general medical setting. The presence and severity of an amnestic syndrome may not be assessable until the acute brain syndrome has subsided. Some idea about progress towards recovery can be made by regular review of these patients every few days. The classical triad of Wernicke's encephalopathy (ataxia, ophthalmoplegia, confusion) may not be present. All toxically confused patients should have Wernicke's encephalopathy considered in their differential diagnoses, and parenteral thiamine supplementation should be part of the routine treatment of toxic confusional states of unknown aetiology, as well as in patients with a definite alcohol-withdrawal delirium.

\section{Intermediate assessment and management}

Once a toxic state has resolved and the patient is consistently alert, a clearer picture of the deficits may be obtained. Several features are thought to be relevant to the development of Korsakoff syndrome and to its recovery.

It may be possible clinically to elucidate various relevant prognostic factors at this stage by careful examination of the patient and by obtaining a collateral history. The collateral history can give information about the rate of onset which may be of prognostic significance.

Classically, the outcome from an episode of Korsakoff syndrome falls roughly into 'quarters'. Approximately $25 \%$ show no recovery, $25 \%$ slight, $25 \%$ significant and the other $25 \%$ show complete recovery from the episode in relation to impairment of recent memory.
At this point assessment needs to be directed towards appropriate placement for rehabilitation. There is an argument for an intermediate admission to appropriate medium-stay/rehabilitation units for a more protracted period of assessment prior to final placement after any recovery has been maximised. This may involve a stay of up to one year.

Arrangements for rehabilitation placement will obviously vary according to local resources. This is currently the topic of some debate and resources may be woefully inadequate in some areas of the country. There is no standard model of a Korsakoff rehabilitation service in use in the UK, although examples exist elsewhere, such as Australia and The Netherlands. There is also debate about which agency is best placed to provide rehabilitation for these patients. Agencies involved include the National Health Service, social work, private (for profit) and voluntary agencies. Different systems may work equally well, but there has been little evaluation of these in practice; we found three studies of relevance here.

One was of a series of 44 patients allocated to nursing homes or specialist sheltered Korsakoff accommodation. Those allocated to nursing homes showed more deterioration in social functioning compared with those allocated to specialist accommodation, although cognitive functioning was equal in both groups (Blansjaar et al, 1992).

The second was a comparison of functioning of patients in ordinary long-stay psychiatric wards compared with those in a specialist Korsakoff rehabilitation ward. At the end of a six-month period, those in the specialised unit showed a significantly greater improvement in the speed of information-processing and improved social behaviour. These improvements were also demonstrated in a group of patients who were subsequently transferred from ordinary long-stay to the specialist unit (Ganzevles et al, 1994).

The third study describes a dedicated in-patient service for this patient group in Sydney, Australia. Of the 104 patients (93 men and 11 women) who underwent a structured rehabilitation programme, $51 \%$ were successfully placed in the community at follow-up 1-2 years after leaving hospital. Of the 53 patients who were successfully placed, the majority were living in boarding houses with supply of meals and a degree of supervision (Lennane,1986).

We would argue that regardless of where rehabilitation services are provided, it is essential for psychiatrists to remain involved to maximise continued coordinated rehabilitative efforts and reassessment. Specialists would also have a role in researching and evaluating the various placements available and this would address the current concerns of bodies such as the Mental Welfare 
Commission about inappropriate detention and placement of these patients.

Once suitable placement has been obtained then a process of multi-disciplinary assessment should begin. This should include review by neuropsychologists, occupational therapists, physiotherapists and appropriate psychiatric personnel. These patients may develop associated psychiatric problems such a psychosis or depressive episodes which may need specific treatment (Lennane, 1986)

A method of classifying these patients that the authors have found useful in clinical practice is the use of the Weschler Adult Intelligence Scale (Revised) (WAIS-R; Wechsler, 1981), for current intelligence quotient (IQ). The National Adult Reading Test (Nelson, 1982) will provide an approximation of the patient's premorbid IQ and the Weschler Memory Scale (Revised) (WMS-R; Wechsler, 1987) indicates the types of memory deficit present and gives a measure of memory quotient (MQ; Jacobsen \& Lishman, 1987). The results of these tests are likely to reveal a range of deficits which allows classification of patients along the two axes of dementia (discrepancy between premorbid IQ and current IQ) and amnesia (discrepancy between IQ and MQ). As we have already stated, this has implications for prognosis in conjunction with information on mode of onset.

Tests of frontal lobe function and behavioural memory tests (Wilson et al, 1985) may be useful as they are likely to highlight more practical day-today problems and may guide rehabilitative efforts and eventual safe permanent placement.

Occupational therapy assessment may include home-based assessments to ascertain whether a return to the home situation with or without support of relatives is feasible. Obviously the presence of supportive carers is an important factor in rehabilitation.

Important in the assessment is whether patients are felt to be at risk of relapse into drinking, particularly if placement in the original home circumstances is being considered. It is a wellrecognised clinical finding that these patients have a drastically reduced tolerance to even small amounts of alcohol and will be at high risk of injury through severe intoxication or collapse. This may result in a further episode of Wernicke-Korsakoff syndrome and impair their final recovery even further (Blansjaar et al, 1992).

\section{Long-term assessment and management}

There is very little literature on this subject. It is known that these patients once stable have a normal life expectancy if abstinent from alcohol, and therefore long-term placement is an expensive and important consideration. Clinical experience and research suggest that after one year, any further recovery is unlikely. However, it is known that adaptation to a new environment can be facilitated by appropriate rehabilitative efforts with emphasis on improving conditioned learning.

\section{Acute treatments}

Intravenous or intramuscular thiamine supplementation is recommended to prevent progression from Wernicke's encephalopathy to Korsakoff syndrome (Cook \& Thomson, 1997). This requires a low threshold for making a presumptive diagnosis in doctors working in accident and emergency, general medical and surgical wards, or any wards where patients are likely to be admitted in a confused state. Patients with head injury, hypothermia, reduced conscious level or toxic confusional state should have Wernicke's encephalopathy considered in their differential diagnosis and be given intravenous or intramuscular thiamine.

The Korsakoff component of the WernickeKorsakoff syndrome associated with alcohol dependence, once established, is thought to be poorly responsive to thiamine replacement treatment, but it is still important to treat the condition aggressively at initial presentation with parenteral vitamins, given the very low incidence of complications of this intervention. Obviously, removal from the drinking environment is also essential, as is adequate treatment of the alcohol-withdrawal syndrome.

\section{Intermediate and long-term treatments}

\section{Specific drug treatment for Korsakoff syndrome}

There have been various attempts to search for pharmacological agents to treat the amnestic syndrome. Agents researched include fluvoxamine, methylphenidate and clonidine. A review of these shows that there is no satisfactory evidence as yet for these agents to be used in routine clinical practice, and further research is required (see Kopelman, 1992; Ferrin, 1994 for reviews). 


\section{Drug treatment of associated psychiatric syndromes}

These patients are at increased risk of various associated psychiatric syndromes (Lennane, 1986). In particular they may develop depression once their cognitive deficits are well-established. The depression may be as a consequence of their organic condition and, in particular, damage to the frontal lobes in those with more global deficits than a pure amnestic syndrome. The depressive syndrome may be worsened by a lack of stimulation or placement in an inappropriate environment, or it may be a response to partial insight into the extent of their deficits. These patients may develop psychotic episodes which again may be worsened by sensory deprivation.

In treating any associated depressive or psychotic symptomatology it is advisable to avoid any drugs with anticholinergic properties. These may worsen the patient's confusion and this may be related to evidence of cholinergic depletion in patients with Korsakoff syndrome, as may occur in those with Alzheimer's disease (Kopelman \& Corn, 1988).

\section{Management of the alcohol problem}

Patients with established amnestic deficits may still have the urge to drink. Others may completely lose this and this will have obvious impact on suitability for various placements. Those with a mild to moderate Korsakoff syndrome are more likely to drink again. It is therefore essential that care is taken to discourage the use of alcohol in these cases. There is evidence that repeated drinking will worsen the cognitive deficit over time.

\section{Cognitive rehabilitation}

There is a large literature on cognitive rehabilitation in general. There is less specific literature on cognitive rehabilitation in alcohol Korsakoff syndrome. Readers interested in cognitive rehabilitation are referred to the Handbook of Memory Disorders (Baddeley et al, 1995). Cognitive rehabilitation encompasses specific techniques to encourage memory rehabilitation, design of environments, use of memory prostheses and styles of nursing used to promote new learning.

Of prime importance is neuropsychological assessment to ascertain the specific cognitive deficits in the patient and the degree of purity of the amnestic disorder. Psychologists may also have a role in doing a behavioural assessment to gain an idea of the patient's functional problems secondary to the memory deficits. These will obviously direct rehabilitative efforts. Succesful memory rehabilitation will depend on the patient's level of motivation, premorbid intellectual functioning and preferred style of interaction.

This is of relevance in that patients with more global alcohol dementia may have more apathy and reduced intellectual functioning, impairing their response to specific memory rehabilitation. Those with a pure amnestic deficit may be more motivated to make up the gap by use of memory prostheses, etc. Another factor impairing response to memory rehabilitation may be unrecognised anxiety or depressive disorders or social isolation and loneliness. This observation has led to development of memory groups by some workers. These can be helpful in reducing anxiety, social isolation or encouraging modelling of new learning techniques.

If the patient has to spend time at home or to have more meaningful encounters with his family and avoid isolation, advice to families as to how to organise information to improve recall may be helpful. This advice can be summarised as: a little and often, checking understanding, encouraging the sufferer to link information and one piece of information at a time. Carers may benefit from contact with a support group. For details of these we refer the reader to Wearing (1992).

\section{Specific techniques in memory rehabilitation in Korsakoff syndrome}

Brooks \& Baddeley (1976) demonstrated that Korsakoff patients are capable of new learning, particularly if information is cued and of certain types. Patients can therefore learn new skills. They showed that even densely amnestic patients can benefit from cueing but have a limited capability of forming new procedural memories, that is, they can demonstrate the acquisition of new verbal and nonverbal skills but do not have the declarative memory capabilities to code the learning of these skills as episodes. Thus, a skill is learned without awareness of the circumstances under which the learning took place, or in fact that it did take place. This has implications for teaching easily measured and significant information to patients, as well as for guiding the utilisation of memory prostheses, in order to maximise patient autonomy.

There is some evidence from single uncontrolled case reports that the use of visual imagery or verbal mediation is superior to rote learning in the 
acquisition of verbal information. It also appears that the visual mode is superior to the verbal mode, but that there are difficulties in getting the patient to generalise information learned. There is also evidence for certain styles of learning being superior.

A study of 16 patients with memory disorder and 16 controls (Baddeley \& Wilson, 1994) showed that 'errorless' was superior to 'errorful' learning. The patients were able to recall more information over a longer period when they were not allowed to guess. An example of this would be a Korsakoff patient who was lost in a ward and asked someone where his room is. In the errorful way of learning the patient would be asked to guess where his room was and would undoubtedly make a mistake. The errorless learning response would have been "I will show you your room. This is your room". By eliminating guessing, the patient avoids making early learning errors which would then be repeated in a perseverative fashion. This has obvious implications for the style of nursing or style of caring by relatives of patients with amnestic disorders. Specific memory rehabilitation and use of prosthetic memories is best promoted in a well-structured environment which reduces stress on memory and promotes new learning.

\section{External memory aids}

A large range of external memory aids is available (see Baddeley et al, 1995). Memory aids can be based on a very simple idea; for example, amnestic patients are fitted with a bleeper which goes off to alert staff if they move too far away from the ward or hostel. Eventually, they would recognise themselves that the bleep means they should turn back.

Other simple memory aids include the making of lists or diaries through to the use of more complex electronic memory aids such as personal organisers or computer programs. There are obvious problems in teaching patients with a specific amnestic syndrome to use these and, again, there is a clear role for involvement of a psychologist. A more recent development is a 'neuropage', which works like an ordinary messenger pager that sends the wearer a message saying, for example, "Take your medication". These devices may be of relevance to people with a milder amnestic deficit living at home or independently. There is a need for further research into which types of memory aid and in what combinations best suit the rehabilitation of Korsakoff patients.

\section{Design of environment}

For some patients with a very severe deficit, the most appropriate way of maximising their independence may lie in the design of their environment. A carefully thought out environment could provide cues to assist orientation and eventual new learning. Examples of these are improving patients' recognition of staff by changing their name badges to make them easily readable and to reflect what nurses are actually called in the ward, rather than their official title. Units should be on the ground floor with large windows to aid patients' orientation for the time of year or time of day. The use of arrows for direction on the floor and colour-coding of rooms and doors would also help orientation. The style of nursing should reflect the research evidence in favour of errorless learning.

\section{Legal issues}

The use of the Mental Health Act may have to be considered with these patients in the early stages of the illness if they are clearly incapable of independent living, uncooperative with treatment and have no adequate family support. At a later stage guardianship provision might need to be utilised in order to move the patient on to the most suitable accommodation. (Data in Scotland show that in the year 1 April 1997 to 31 March 1998, out of 123 guardianships granted, 20 were for patients with alcohol-related brain damage; A. Jacques, personal communication.) Additional legal measures may sometimes have to be considered in order to manage patients' financial affairs on their behalf. Restrictions on personal finances can be effective in limiting further misuse of alcohol in order to allow some patients a degree of freedom to come and go when well-established in a particular placement.

\section{Conclusions}

In view of the above the authors contest that it is the duty of the psychiatrist to regularly review these patients to coordinate special assessment and to ensure a placement that maximises the patients' independence within the limitations of their residual disabilities (see Box 3). In other words:

\footnotetext{
"The eventual disposal of the patient to his family, nursing home or mental institution is undertaken on the basis of the severity of the amnestic psychosis as well as the existing family and social circumstances, but only after one is certain that no further improvement in memory function is possible." (Victor et al, 1989).

Availability of some of the more specialised rehabilitative techniques is limited in our current
} 
Box 3. Rehabilitative principles

Regular review required in first year after diagnosis

Eventual placement determined by careful multi-disciplinary assessment

If patient not capable of independent living, then provisions of Mental Health Act and guardianship may have to be utilised

Social functioning can be improved with the use of memory rehabilitation techniques

Design of environment to maximise independent living

Use whatever means possible to deter further use of alcohol

health care system and there seems to be a case for the development of specialist provision on a regional basis in areas where the condition is prevalent. At least two health boards in Scotland are currently looking at this possibility. There is a small literature from Australia and The Netherlands suggesting that such specialised provision can benefit these patients in terms of social functioning.

\section{References}

Anonymous (1987) Memory testing: no thermometers available Lancet ii, 604-605.

Baddeley, A. D. \& Wilson, B. A. (1994) When implicit learning fails: amnesia and the problem of error elimination. Neuropsychologia, 32, 53-68.

-, Wilson, B. A. \& Watts, F, N. (eds) (1995) Handbook of Memory Disorders. Chichester: John Wiley and Sons.

Blansjaar, B. A., Takens, H. \& Zwinderman, A. H. (1992) The course of alcohol amnestic disorder: a three-year follow-up study of clinical signs and social disabilities. Acta Psychiatrica Scandinavica, 86, 240-246

Brooks, D. N. \& Baddeley, A. D., (1976) What can amnesic patients learn? Neuropsychologia, 14, 111-122.

Cook, C. C. H. \& Thomson A. D. (1997) Review: B complex vitamins in the prophylaxis and treatment of WernickeKorsakoff syndrome. British Journal of Hospital Medicine, 57, 461-465.

Cutting, J. (1978) The relationship between Korsakov's syndrome and 'alcoholic dementia'. British Journal of Psychiatry, 132, 240-251.

Ferrin, J. M. (1994) Drug efficacy in the treatment of Wernicke and Korsakoff syndromes. Journal of Applied Rehabilitation Counselling, 25, 32-36.

Ganzevles, P. G. J., De Geus, B. W. J. \& Wester, A. J. (1994) Cognitive and behavioural aspects of Korsakoff's syndrome: The effect of special Korsakoff wards in a general psychiatric hospital. Tijdschrift voor Alcohol, Drugs en Andere Psychotrope Stoffen, 20, 20-31.

Jacobson, R. R. \& Lishman, W. A. (1987) Selective memory loss and global intellectual deficits in alcoholic Korsakoff's syndrome. Psychological Medicine, 17, 649-655.

Kopelman, M. D. (1992) The psychopharmacology of human memory disorders. In Clinical Management of Memory Problems (2nd edn) (eds B. A. Wilson \& N. Moffat), pp. 189-215. London: Chapman \& Hall.
- (1995) The Korsakoff syndrome. British Journal of Psychiatry, 166, 154-173.

— \& Corn, T. H. (1988) Cholinergic 'blockade' as a model of comparison of the memory deficits with those of Alzheimer type dementia and the alcoholic Korsakoff syndrome. Brain, 111, 1079-1110.

Kok, A. F. (1991) Developments in the care of Korsakoff patients. Tijdschrift voor Alcohol, Drugs en Andere Psychotopic Stoffen, 17, 3-9.

Lennane, K. J. (1986) Management of moderate to severe alcohol-related brain damage (Korsakoff's syndrome). Medical Journal of Australia, 145, 136-143.

Lishman, W. A. (1990) Alcohol and the brain. British Journal of Psychiatry, 156, 635-644.

Nelson, H. E. (1982) National Adult Reading Test (NART) for the Assessment of Premorbid Intelligence in Patients with Dementia: Test Manual. Windsor: NFER-Nelson.

Price, J. (1985) The Wernicke-Korsakoff syndrome in Queensland, Australia: antecedents and prevention. Alcohol and Alcoholism, 20, 233-242.

Ramayya, A. \& Jauhar, P. (1997) Increasing incidence of Korsakoff's psychosis in the East End of Glasgow. Alcohol and Alcoholism, 32, 281-285.

Victor, M., Adams, R. D. \& Collins, G. H. (1971) The WernickeKorsakoff Syndrome and Related Neurological disorders Due to Alcoholism and Malnutrition (1st edn). Philadelphia, PA: F. A. Davis.

- - \& - (1989) The Wernicke-Korsakoff Syndrome and Related Neurological Disorders due to Alcoholism And Malnutrition (2nd edn). Philadelphia, PA: F. A. Davis.

Wearing, D. (1992) Self help groups. In Clinical Management of Memory Problems (2nd edn) (eds B. A. Wilson \& N. Moffat), pp. 271-304. London: Chapman \& Hall.

Wechsler, D. (1981) WAIS-R Manual. New York: Psychological Corporation.

Wechsler, D. (1987) WMS-R Manual. New York: Psychological Corporation.

Wilson, B. A., Cockburn, J. \& Baddeley, A. (1985) Rivermead Behavioural Memory Test. Bury St Edmunds: Thames Valley Test Company.

\section{Multiple choice questions}

1. Recovery from alcohol Korsakoff syndrome (alcohol amnestic syndrome) is:

a never seen

b unlikely if the condition is still present after a year

c more likely with sudden onset cases

d seen to some degree in the majority

e more or less complete in one-fifth to one-quarter of cases.

2. Alcohol Korsakoff syndrome is:

a always preceded by Wernicke's encephalopathy

b a progressive neurodegenerative disorder with decreased life expectancy, given abstinence from alcohol

c characterised by a deficit in remote memory with intact recent memory

d readily ameliorated with pharmacotherapy

e increasing in prevalence in some European countries. 
3. Wernicke's encephalopathy:

a is most commonly associated with alcohol dependence in Western countries

$b$ is due to deficiency of thiamine (vitamin B1)

c should be treated initially with oral thiamine

d is only present whan all three classical features are seen (ophthalmoplegia, ataxia, confusion)

e can be present along with alcohol-withdrawal delirium (delirium tremens).

4. The rehabilitation of individuals with alcohol Korsakoff syndrome is:

a best served in general, non-specialised units

b not possible as they have no capacity for new learning

c enhanced by the use of memory aids

d based on principles of 'errorful' learning

e necessary to maximise social functioning.
5. The following may be useful in the management of this patient group:

a guardianship

b high doses of antipsychotic drugs with anticholinergic properties

c treatment of associated depressive illness

d continuity of psychiatric involvement

e support services for carers.

\section{MCQ answers}

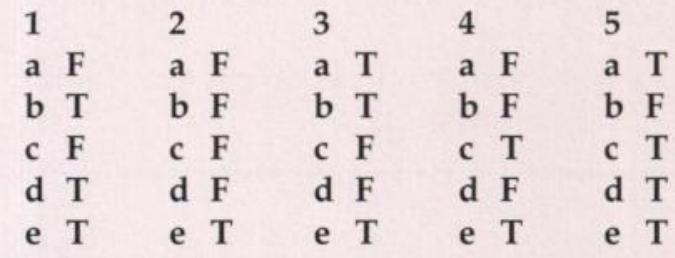

\section{Royal College of Psychiatrists}

\section{World Congress of Psychiatrists, Hamburg 6-11 August 1999}

Gaskell Press imprint of the Royal College of Psychiatrists will be displaying and selling publications at this year's congress. There will be leaflets and catalogues to collect and publications in stock to purchase. The following will be launched at this year's meeting:

- Promoting Mental Health Internationally

- $\quad$ Getting the Message Across (Focus on Dissemination)

- Mental Health of Ethnic Minorities: An Annotated Bibliography

- $\quad$ Evidence-Base Briefing: Dementia

- Improving the Care of Elderly People with Mental Health Problems: Clinical Audit Project Examples 